A puntes del CENES

ISSN 0120-3053

Volumen 30 - №. 52

Segundo Semestre 2011

Págs. 59 - 86

\title{
Evidencia empírica de la teoría del consumo para Colombia 2000-2010*
}

\section{Empirical evidence of the theory of consumption for colombia 2000-2010}

\author{
Julián Augusto Casas Herrera** \\ José M auricio Gil León****
}

Fecha de recepción: 27 de marzo de 2011

F echa de aprobación: 30 mayo de 2011

\footnotetext{
* Este documento surge de la integración de los grupos de investigación, Modelos de Desarrollo en América Latina (MODEAL) y Observatorio de Coyuntura Económica Regional y Urbana (OIKOS), de la Escuela de Economía de la Universidad Pedagógica y Tecnológica de Colombia (UPTC), específicamente de los jóvenes investigadores Julián A. Casas H. y J. Mauricio Gil L. Ambos agradecen a COLCIENCIAS su apoyo.

** Economista Universidad Pedagógica y Tecnológica de Colombia, UPTC. Actualmente se desempeña como docente de la UPTC (Tunja, Colombia). Asimismo es joven investigador de COLCIENCIAS. Correo electrónico: julicasher84@yahoo.es

*** Economista Universidad Pedagógica y Tecnológica de Colombia, UPTC. Actualmente se desempeña como docente de la UPTC (Tunja, Colombia). Igualmente es joven investigador de COLCIENCIAS. Correo electrónico: mauricio8827@hotmail.com
} 


\section{Resumen}

En este trabajo se evalúan las hipótesis de consumo de Keynes, Friedman, M odigliani y Hall, mostrando sus elementos teóricos, junto con la verificación econométrica de cada una para Colombia, con datos trimestrales, en el periodo 2000-2010. Se muestran las diferencias que se pueden obtener en el análisis de estas, con sus respectivos impactos en la política económica. En el texto se concluye que la hipótesis keynesiana es útil para un estudio de política económica a corto plazo; mientras que la de Friedman, con expectativas adaptativas, resultó, más consistente a largo plazo que la de M odigliani y la de Hall.

Palabras clave: teorías del consumo, función keynesiana, ciclo vital, ingreso permanente, caminata aleatoria.

Clasificación JEL: B21, B22, B23, E21.

\section{Abstract}

The paper evaluates the hypothesis of consumption of Keynes, Friedman, Modigliani and Hall, showing its theoretical elements along with the econometric verification of each one for Colombia, with quarterly data from the period 2000 to 2010 . The differences that can be obtained in their analysis are shown with their consequent impacts on economic policy. The article concludes that the Keynesian hypothesis is useful for an analysis of economic policy in the short term; while Friedman's hypothesis, with adaptive expectations, was more consistent in the long run than that of Modigliani and Hall.

Keywords: consumption theories, Keynesian function, life cycle, permanent income, random walk.

JEL Clasification: B21, B22, B23, E21. 


\section{Introducción}

Dada la importancia del consumo de los hogares dentro del Producto Interno Bruto (PIB) de Colombia, que en el periodo comprendido entre el 2000 y 2010 representó en promedio un $66.41 \%$, según información suministrada por el DANE, este documento establece, a la luz de algunos enfoques teóricos sobre la teoría del consumo -función keynesiana, ciclo vital, hipótesis de Hall y renta permanente, una estimación de los parámetros a los que hacen referencia estos enfoques para la economía colombiana, con el fin de establecer algunos determinantes del consumo agregado.

Esto adquiere importancia en la medida que los análisis que se desprenden alrededor del consumo conciernen a la elaboración de medidas de política económica conducentes a afectar la demanda agregada y, por ende, la tasa de crecimiento económica. Por ejemplo, la crisis económica desatada desde 2008 en los Estados Unidos y en algunos países de Europa, ha llevado a tomar distintas medidas con el propósito de reactivar el crecimiento económico. Entre las medidas que se proponen se encuentran disminuir el gasto público o aumentar los impuestos, lo cual significa aumentar el ahorro agregado y, en consecuencia, reducir el consumo agregado. Por tanto, se considera que el tema que se desarrolla en las próximas líneas es de actualidad.

No obstante, cabe señalar que el documento solo intenta verificar empíricamente, con ayuda de la econometría y sus pruebas, la teoría económica que subyace en estos enfoques. Lo anterior podría dar pie a un 
severo ataque al texto, puesto que se estaría dejando de lado el interesante debate que se presentó con el surgimiento de cada enfoque. Sin embargo, orientar el documento en el sentido de mostrar el rico debate que se presentó entre diversos autores precisaría de otro tipo de documento, que se sale del dominio que plantea este texto: mostrar, por un lado, la significancia estadística de los resultados; y por el otro, revelar la significancia económica de cada resultado como insumo para el diseño de medidas de política económica.

Al hacer este ejercicio, se encontró que la función de consumo keynesiana suministra la propensión marginal a consumir y la propensión marginal a ahorrar a corto plazo; mientras que la hipótesis del ingreso permanente provee la propensión marginal a consumir y la propensión marginal a ahorrar a largo plazo. Además, se llega a la conclusión que en Colombia se cumple, que a medida que crece el ingreso disponible, los colombianos dedican una mayor proporción al ahorro, lo cual tiene importantes repercusiones en la tasa de crecimiento económico, a largo plazo, de la economía colombiana.

Por último, para llegar a esa conclusión, el documento se dividió en seis secciones contando esta introducción como la primera. La segunda señala los planteamientos teóricos del enfoque de consumo keynesiano, junto con la estimación econométrica para la economía colombiana. La tercera muestra la teoría de la renta permanente de Friedman, así como la estimación econométrica de la función planteada por este autor. La cuarta enuncia los aspectos teóricos más relevantes de la teoría del ciclo vital de Modigliani, junto con la estimación econométrica. En la quinta se muestra la hipótesis de Hall y la respectiva estimación econométrica. Finalmente, se presentan las conclusiones del documento.

\section{Hipótesis de consumo keynesiana}

\subsection{Revisión teórica}

Los aportes del economista inglés John Maynard Keynes surgen en una época en la que el problema por solucionar era el creciente desempleo. En ese sentido, la motivación del trabajo de Keynes radicaba en entender qué y cómo funciona el nivel de empleo en una economía. Para responder a estos interrogantes, él identificó que los componentes más significativos que inciden en la dinámica del empleo son la inversión y el consumo (Keynes, 1936).

Keynes señaló que la inversión es el componente más volátil, mientras que el consumo tiene un comportamiento relativamente estable. A partir de esto, él ideó una teoría en la que la variable clave es el consumo, debido a que es la que otorga mayor dinamismo a la demanda 
agregada por ser el componente que tiene mayor participación dentro de la misma ${ }^{1}$.

El trabajo de Keynes sitúa al consumo como una función no lineal, en la que identifica la existencia de varios factores determinantes del consumo que brindan la explicación al comportamiento de la propensión marginal a consumir (PMC). Los factores que escoge Keynes (1936) son objetivos y subjetivos; dentro de los primeros se encuentran: "el cambio en la unidad de salario, un cambio en la diferencia entre ingreso e ingreso neto ${ }^{2}$, los cambios imprevistos en el valor de los bienes de capital ${ }^{3}$, cambios en la tasa de descuento del futuro ${ }^{4}$, cambios en la política fiscal, y cambios en las expectativas acerca de la relación entre el nivel presente y el futuro de ingreso". También Keynes (1936) señala que existen algunos factores subjetivos que explican por qué abstenerse de gastar sus ingresos, tales como:

Formar reservas para contingencias imprevistas; proveer para una anticipada relación futura entre el ingreso y las necesidades del individuo y su familia, diferente a la existente en la actualidad; gozar del interés y la apreciación; disfrutar del gasto gradualmente creciente; disfrutar de una sensación de independencia; amasar recursos para proyectos especulativos o de negocios; legar una fortuna; o satisfacer la propia avaricia ${ }^{5}$.

Sin embargo, debido a que Keynes (1936) plantea la estabilidad en la función consumo, dice que este gasto depende del volumen del ingreso total, y deja los cambios en la PMC como secundarios. En ese sentido, es posible basarse en la ley psicológica fundamental, que señala que un aumento del ingreso genera un aumento en el consumo, aunque no en la misma proporción del ingreso. Por tanto, según Keynes (1936), el incremento en los ingresos se acompaña de un incremento mayor en el ahorro, es decir,un nivel absoluto mayor de ingreso ampliará la brecha entre ingreso y consumo, “(...) porque la satisfacción de las necesidades primarias inmediatas de un hombre y su familia es, un motivo más fuerte que los relativos a la acumulación, que sólo adquieren predominio efectivo cuando se ha alcanzado cierto margen de comodidad". En síntesis, la función consumo tradicional keynesiana viene definida como no lineal, porque su medición está predispuesta a cualquier modificación de tipo subjetivo por parte del individuo.

\footnotetext{
En promedio, para las economías del mundo, el consumo representa entre un medio a dos terceras partes de la demanda agregada (Fernández, 2009).

2 El ingreso neto se refiere al consumo más la inversión neta, es decir, lo que el agente consume más lo que ahorra y lo destina a la inversión, descontándole, a la vez, la depreciación del capital.

3 Esto incide en el consumo de las clases propietarias de riqueza, porque los agentes son sensibles a los cambios imprevistos en el valor monetario de la riqueza.

4 Tiene en cuenta los cambios inesperados del poder adquisitivo del dinero, y de los riesgos de no gozar de los bienes futuros.

5 Keynes lo describe como el acto irracional de inhibirse de gastar.
} 
Ahora, la no linealidad de la función de consumo keynesiana es cambiada por la noción de Hansen y Hicks (1989) que establecen una relación lineal entre el consumo y el ingreso disponible, es decir, integran todos los factores en este ingreso. Entonces, la función de consumo queda constituida por un parámetro autónomo $(\alpha)$, un segundo parámetro $(\beta)$ y el ingreso disponible (YD) (Fernández, 2009). El primer parámetro señala que el consumo es independiente del ingreso disponible, es decir, aquel consumo que existiría, así el ingreso disponible disminuya o aumente ${ }^{6}$. El segundo parámetro se ha denominado PMC, indicando la porción adicional que cada agente ${ }^{7}$ asigna del aumento de su YD para consumir, ésta necesariamente debe tener un valor entre cero a uno ${ }^{8}$. Por último, el YD se define como el ingreso total $(Y)$ menos los impuestos $(T)$. En términos matemáticos, la función linealizada de Keynes, que fue reformulada por Hicks y Hansen, es la siguiente:

$$
\begin{gathered}
C=\alpha+\beta Y D \\
0<\beta<1
\end{gathered}
$$

Cabe señalar que existen tres conjeturas a partir de la forma funcional de la ecuación (1). La primera indica que el consumo depende fundamentalmente del ingreso disponible. La segunda establece que los agentes acrecentarían su consumo si el ingreso aumenta, pero no en la misma medida del incremento del ingreso. La última conjetura manifiesta que a medida que crece el ingreso en el tiempo, los agentes dedicarán una mayor parte del ingreso hacia el ahorro (Hernández \& Chahín, 2008).

Según Hernández y Chahín (2008), de estas tres conjeturas las dos primeras se cumplen para Colombia, mientras que la tercera no se cumple puesto que la evidencia muestra que la tasa de ahorro permanece constante en el tiempo. Esto tiene connotaciones de tipo político, puesto que la función de Keynes sostiene que basta un aumento del ingreso disponible para incrementar el consumo.

\subsection{Estimación econométrica}

Para estimar la función de consumo keynesiana se usó la metodología de Blanchard (2003), donde se toma la variación del consumo de un periodo respecto a la variación entre periodos del ingreso disponible (YD) separando en ambas variables la variación media ${ }^{9}$. Este método es necesario porque retira el efecto

$6 \quad$ La lógica de este suceso radica en que los agentes, aunque vean disminuido su ingreso disponible, necesariamente, harán todo lo posible por consumir, posiblemente pidiendo prestado o desahorrando. Esto implica que este parámetro asume un valor positivo.

7 Las diferentes teorías del consumo asumen que el agente del cual se está hablando es el denominado agente representativo. No obstante, el lector interesado puede encontrar en Clarida (1991) y Kirman (1992) la propuesta de abandonar esta figura.

8 Esto implica que el porcentaje del ingreso disponible que no se consume se ahorra. Esto se denomina la propensión marginal a ahorrar.

9 Para que quede más clara la metodología empleada, se pone el siguiente ejemplo: si la diferencia entre un trimestre y otro es de 10 millones y el promedio muestral de las diferencias de un trimestre a otro es de 7 millones, el dato que se toma en la metodología sería 3 (10-7). 
de los incrementos tendenciales, lo cual tiene como ventaja estimar la PMC real de corto plazo y evitar la sobreestimación de este parámetro.

Blanchard (2003) sugiere que para la estimación de la PMC es posible utilizar rezagos, ya que en economía lo común es encontrar retardos en los ajustes de las variables. Por tanto, cuando se trata de estimar el parámetro que define la PMC, es necesario precisar que este no se encuentra para un único periodo sino para varios, y el número de rezagos se establecería con un criterio de grado de ajuste estadístico.

La estimación de la PMC para Colombia se hace utilizando datos trimestrales del DANE a precios de 2005 y desestacionalizados, entre el primer trimestre del 2000 hasta el cuarto trimestre de 2010, del consumo agregado de los hogares $(\mathrm{CH})$ y del PIB, menos el valor de los impuestos pagados que viene a representar el YD. De este modo, se pasa a estimar la función de consumo keynesiana para Colombia por medio del método de mínimos cuadrados ordinarios (MCO), ya que este ofrece los coeficientes estructurales de la función lo que unido al cumplimiento de los supuestos, permitirá reducir los errores cuadráticos, y en consecuencia, tener estimadores útiles para propósitos de política económica. La ecuación que se estimó es la siguiente:

$D M C H_{\mathrm{t}}=\beta_{1} D M Y D_{\mathrm{t}}+\beta_{2} D M Y D_{\mathrm{t}-1}+\mu_{\mathrm{t}}$

Donde DMCH es la diferencia de $\mathrm{CH}$ de un trimestre a otro, menos el promedio de los cambios entre trimestres de $\mathrm{CH}, \mathrm{y}$ DMYD es la diferencia de YD de un trimestre a otro, menos el promedio de los cambios entre trimestres de YD. Además, la suma de ambos parámetros indicará la PMC. Los resultados se muestran en el Tabla 1.

Tabla 1. Estimación de la función de consumo keynesiana

\begin{tabular}{|l|c|c|c|r|}
\hline \multicolumn{4}{|c|}{ Variable Dependiente: DMCH } \\
\hline \multicolumn{4}{|c|}{ Método: MCO } \\
\hline \multicolumn{4}{|c|}{ Muestra: 2000-I 2010-IV } \\
\hline Vobervaciones: 42 después de ajustado \\
\hline Variable & Coeficiente & Desv. Estand & t-Estad & 0.0000 \\
DMYD & 0.382917 & 0.069606 & 5.501234 & 0.0016 \\
DMYD(-1) & 0.238122 & 0.070376 & 3.383583 & 4.759157 \\
\hline R-cuadrado & 0.480372 & \multicolumn{2}{|c|}{ Media var depend } & 487.8555 \\
R-cuadrado ajustado & 0.467381 & Desv. Estand var depend & 14.71716 \\
Desv. Estand de reg & $3.56 E+02$ & Criterio Schwarz & 1.729064 \\
SRC & $5.07 E+06$ & Durbin-Watson & 0.3607 \\
Prob. Jarque-Bera & 0.837837 & Breusch-Pagan-Godfrey & 0.8302 \\
Prueba de Klein & NA & Test de Ramsey-Prob & \\
\hline
\end{tabular}

Fuente: cálculos propios en eviews 6.0 
En el Tabla 1 se evidencia el cumplimiento de los supuestos de normalidad (JarqueBera), homoscedasticidad (Breusch-PaganGodfrey), no autocorrelación (DurbinWatson) y correcta especificación (test de Ramsey). Además, la bondad del ajuste de la línea regresión es de $48 \%$, que en términos económicos señala que el YD del periodo t y $\mathrm{t}-1$ explican el comportamiento del $\mathrm{CH}$ en el periodo $\mathrm{t}$ en el porcentaje indicado. Finalmente, se muestra que todos los coeficientes son significativos a un nivel de confianza del $90 \%{ }^{10}$.

La PMC estimada para Colombia en el periodo muestral fue de 0.62 , teniendo en cuenta que el efecto de una variación del YD en un peso provoca que el $\mathrm{CH}$ responda en el mismo periodo en 0.38 pesos y el siguiente en 0.24 pesos. Este resultado contrasta con la estimación del documento para el Plan Nacional de Desarrollo (20102014) elaborado por Briceño y Camelo (2011) que presenta una estimación de la PMC para Colombia de 0.63. Esta última estimación podría estar sobrestimando el impacto del YD de los colombianos sobre el consumo, lo cual podría tener efectos de política económica a la hora de sacar conclusiones alrededor de la tasa de ahorro del país.

\section{Hipótesis del ingreso permanente}

\subsection{Revisión teórica}

Milton Friedman, en 1957, formuló la hipótesis del ingreso permanente (HIP) para explicar la conducta del consumidor, fundamentándose en la teoría de consumo de Irving Fisher ${ }^{11}$ para señalar que el consumo no solo depende de la renta corriente, sino que manifestó que ante variaciones en los ingresos de los consumidores, estos desconocen si los cambios en los ingresos son transitorios o permanentes. Conocimiento que resulta vital, dado que los consumidores no reaccionarán de la misma manera frente a un cambio transitorio que ante uno permanente.

Friedman señaló que el consumo, a diferencia de Keynes, no está determinado solo por el ingreso corriente sino por todos los ingresos que reciban los consumidores a lo largo de sus vidas. Por ello, la HIP centra su atención en la distinción entre consumo permanente y consumo corriente, así como entre ingreso permanente e ingreso corriente, dado que el modelo dice que los agentes consumen no de acuerdo con sus ingresos actuales sino de acuerdo con los ingresos que esperan recibir a lo largo de su vida.

En ese sentido, Friedman afirmó que el ingreso tiene dos componentes: uno permanente y otro transitorio. El primero refleja el efecto de los factores considerados como permanentes, tales como la riqueza no humana, los atributos personales como la preparación, las habilidades y la personalidad, la ubicación de la actividad

\footnotetext{
$\overline{10}$ Se aclara que en todo el trabajo se va a tener este nivel de confiabilidad.

11 En 1953, Fisher desarrolló un modelo de consumo intertemporal que analiza cómo los consumidores toman sus decisiones frente al consumo y el ahorro.
} 
económica, entre otras. El segundo muestra sucesos transitorios tales como eventos accidentales, los ciclos económicos, en síntesis refleja la incertidumbre (Fernández, 2009). Siguiendo la formulación de Fernández (2009), se llega a las siguientes expresiones para definir la HIP.

$$
\begin{aligned}
& C^{p}=k(r, A, u) \mathrm{y}^{p} \\
& y=y^{p}+y^{t} \\
& c=c^{p}+c^{t}
\end{aligned}
$$

El consumo permanente $\left(C^{p}\right)$ está en función de la tasa de interés $(r)$, de la riqueza $(A)$ y de los gustos del consumidor $(u)$. Por tanto, la ecuación 3 refleja la relación entre el ingreso permanente y el consumo permanente, donde $\mathrm{k}$ es la propensión marginal a consumir, siendo esta constante con respecto al ingreso permanente $\left(y^{p}\right)$, aunque esta propensión se puede desviar cuando algún supuesto se incumpla. La ecuación 4 muestra que el ingreso $(y)$ es resultado de la suma del ingreso permanente $\left(y^{p}\right)$ y del ingreso transitorio $\left(y^{t}\right)^{12}$. Por último, la ecuación 5 muestra que el consumo $(c)$ es resultado de la suma del consumo permanente $\left(c^{p}\right)$ y del consumo transitorio $\left(c^{t}\right)$.

El modelo de la HIP supone expectativas adaptativas, donde los agentes las están actualizando de acuerdo con el pasado más reciente. En consecuencia, la ecuación que define el consumo de acuerdo con la renta permanente queda constituida como:

$y_{t}^{P}=r \varphi\left(y_{t}+\varphi y_{t-1}+\varphi y_{t-2}+\ldots \varphi^{\mathrm{n}} y_{t-n}\right)$

La ecuación (6) representa la HIP, donde $\varphi=1 /(1+r)$ representa el factor de descuento que lleva a valor presente los ingresos actuales y pasados mediante una aproximación con una progresión geométrica decreciente del promedio ponderado de los valores. Por otra parte, se debe advertir que dada la existencia de choques (transitorios o permanentes), los consumidores reaccionan de manera distinta puesto que se supone que los permanentes son más fuertes que los primeros.

Además, en la HIP se argumenta que el consumo se planifica de acuerdo con los ingresos esperados a lo largo de la vida, asunto diferente al análisis keynesiano, que postula la relación con el ingreso disponible. Según la explicación teórica dada en un comienzo lo que le interesa a este modelo es establecer cuál es el comportamiento del ingreso permanente ante cambios en el ingreso corriente.

En consecuencia, para hacerlo se supone que el ingreso permanente de los hogares se ajusta con un rezago a los

\footnotetext{
12 Dado que el ingreso corriente $\left(y_{t}\right)$ es la suma del ingreso permanente $\left(y^{P}\right)$ y del ingreso transitorio $\left(y^{T}\right)$, se debe advertir que el $y^{P}$ es la parte del total del ingreso que los consumidores esperan recibir a lo largo de su vida; en cambio, el $y^{T}$ es algo que no esperan los consumidores en su futuro. Por eso, Friedman concluye que el ingreso transitorio no afecta el consumo.
} 
cambios en la renta corriente, ya que en este modelo se debe imponer el supuesto de expectativas adaptativas de los hogares (Roca, 2008). Para ello el modelo parte de la ecuación (6), y llega a la siguiente:

$$
y_{t}^{P}-y_{t-1}^{P}=\delta\left[y d_{t}-y_{t-1}^{P}\right]
$$

La ecuación (7) señala que el ingreso permanente de los hogares se ajusta con un rezago a las fluctuaciones del ingreso corriente -esto por la hipótesis de expectativas adaptativas-. Además, $\delta$ es un parámetro de expectativas, que refleja el valor que regula el ajuste. Este presenta la siguiente restricción: $0<\delta<1$. Desarrollando se tiene:

$y_{t}^{P}=\frac{\delta}{1-(1-\delta) L} y d_{t}$

Reespecificando la ecuación (3) se tiene:

$c_{\mathrm{t}}=\beta_{1}+\beta_{2} y d+\beta_{3} A_{\mathrm{t}-1}+\beta_{4} r_{\mathrm{t}}$

Incluyendo el supuesto de las expectativas a la función lineal de consumo, es decir, se sustituye (8) en (9), y se tiene:

$$
\begin{aligned}
& {[1-(1-\delta) L] c_{\mathrm{t}}=\beta_{1}[1-(1-\delta) L]+\beta_{2} \delta y d_{t}} \\
& +\beta_{3}[1-(1-\delta) L] A_{\mathrm{t}-1}+\beta_{4}[1-(1-\delta) L]
\end{aligned}
$$

Reordenando resulta:

$$
\begin{aligned}
& c_{\mathrm{t}}=\delta \beta_{1}+(1-\delta) c_{\mathrm{t}-1}+\beta_{2} \delta y d_{t}+\beta_{3} A_{\mathrm{t}-1} \\
& +\beta_{3}(\delta-1) A_{\mathrm{t}-2}+\beta_{4} r t+\beta_{4}(\delta-1) r_{\mathrm{t}-1}
\end{aligned}
$$

La ecuación (11) es la que muestra todo el fundamento de la HIP con expectativas adaptativas ${ }^{14}$. En síntesis, Friedman expresa que las fluctuaciones en el ingreso transitorio no tienen repercusiones significativas en el consumo futuro de los consumidores. No obstante, el ingreso transitorio adquiere relevancia dentro de las elecciones de ahorro de los individuos. De modo que si este es positivo, se espera que el ahorro también lo sea, y viceversa. Así que los consumidores acuden al ahorro para mantener un nivel de consumo estable a lo largo de sus vidas ${ }^{15}$.

\subsection{Estimación econométrica}

Para la estimación de la HIP se tomaron las mismas variables de la función de consumo keynesiana ( $\mathrm{CH}$ y YD), junto con la riqueza de los hogares (A) representada con el Índice Precios de la Vivienda Usada (IPVU) que es calculado por el Banco de la República con base $1990=100 \mathrm{y}$, en este documento, es tomado en términos reales ${ }^{16}-; y$ finalmente, para tener una explicación

13 Donde $L$ representa el operador de rezagos.

14 Ver Cagan (1956) quien desarrolló la hipótesis de las expectativas adaptativas que señala que los agentes económicos hacen sus pronósticos sobre el futuro teniendo en cuenta el pasado.

15 Recuérdese que el ahorro representa un consumo futuro.

16 La justificación de tomar esta variable como proxy de la riqueza se debe a que en Colombia, según cifras dadas en el estudio de López y Salamanca (2009), la vivienda representó en el año 2000 el 71 \% de la riqueza de las familias. 
más sólida se interviene la restricción de la liquidez, que se refiere a las restricciones crediticias que tienen los consumidores al momento de tomar sus decisiones de gasto. En este sentido existen estudios realizados para EE.UU. como el de Flavin (1985) y el de Gross y Nicholas (2001), que identifican la importancia del crédito para el consumo de los hogares. En la estimación se toma el crédito para el sector privado (CRED) a precios de 2005.

Utilizando el método de MCO se identifica que las variables fundamentales para explicar el modelo de consumo con HIP son: el consumo rezagado un trimestre, la sumatoria de los rezagos del ingreso disponible, el valor de la riqueza y la restricción a la liquidez. Por tanto, la ecuación formada para la estimación es la siguiente:

$$
\begin{aligned}
C H_{t}= & \delta \beta_{1}+(1-\delta) C H_{t-1}+\beta_{2} \delta \quad Y D_{t} \\
& +\beta_{3} \mathrm{~A}_{t}+\beta_{4} C R E D_{t}+\mu_{t}
\end{aligned}
$$

La ecuación anterior muestra el modelo econométrico que se estima, donde los errores de la estimación reflejan un comportamiento $\mu_{t}=\varepsilon_{t}-(1-\delta) \varepsilon_{t-1}$ que explica como los errores se van ajustando en el tiempo de acuerdo con el parámetro de expectativas. Ahora, como se quiere identificar el efecto a lo largo del tiempo, se continúa utilizando la metodología de
Blanchard (2003), y esto se muestra en la siguiente ecuación:

$$
\begin{gathered}
D M C H_{\mathrm{t}}=\rho_{1} D M C H_{\mathrm{t}-1}+\rho_{2} D M Y D_{\mathrm{t}}+ \\
\rho_{3} D M Y D_{\mathrm{t}-1}+\rho_{4} D M A_{\mathrm{t}}+\rho_{5} D M C R E D_{\mathrm{t}}+\mu_{\mathrm{t}}
\end{gathered}
$$

Antes de hacer la estimación de la ecuación (13) es necesario probar la relación a largo plazo entre el grupo de variables en niveles $\mathrm{CH}$ frente a la A y $\mathrm{CH}$ frente a CRED. La prueba realizada es la de Johansen (ver Tabla 2), que define que en cada variable existe a lo más una relación de equilibrio a largo plazo.

Establecida la cointegración de las series es posible efectuar la estimación econométrica. Ahora es necesario definir la prueba de diagnóstico mediante la evaluación del cumplimiento de los supuestos de la estimación, donde se cumplen los supuestos de MCO. Asimismo, la bondad del ajuste es de $62.9 \%$ (ver Tabla 3), es decir, todas las variables tomadas explican en este porcentaje al $\mathrm{CH}$, que en términos comparativos es $15 \%$ mayor a la función keynesiana. Con esto es posible interpretar los resultados, aunque es necesario transformar los coeficientes con las siguientes formulas:

$$
\begin{gathered}
\delta=1-\rho_{2} ; \beta_{1}=\left(\rho_{2}+\rho_{3}\right) / \delta ; \\
\beta_{2}=\rho_{4} ; \beta_{3}=\rho_{5}
\end{gathered}
$$


Tabla 2. Prueba de cointegración para CH, A, CRED

\begin{tabular}{|c|c|c|c|c|}
\hline \multicolumn{5}{|c|}{ PRUEBA DE JOHANSEN } \\
\hline \multicolumn{5}{|c|}{ Tendencia Lineal Determinística } \\
\hline \multicolumn{5}{|c|}{ 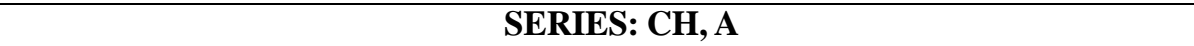 } \\
\hline \multirow{2}{*}{\multicolumn{5}{|c|}{ Muestra: 2000-I 2010-IV }} \\
\hline & & & & observaciones: 42 después de ajustado \\
\hline Hipótesis nula & Eigenvalue & $\begin{array}{l}\text { Traza } \\
\text { estad }\end{array}$ & $\begin{array}{c}\text { Valor Crítico } \\
0.05\end{array}$ & Prob. \\
\hline Ninguna & 0.438407 & 24.81098 & 15.49471 & 0.0015 \\
\hline A lo más 1 & 0.013665 & 0.577872 & 3.841466 & 0.4471 \\
\hline \multicolumn{5}{|c|}{ SERIES: CH CRED } \\
\hline \multicolumn{5}{|c|}{ Muestra: 2000-I 2010-IV } \\
\hline \multicolumn{5}{|c|}{ observaciones: $\mathbf{4 2}$ después de ajustado } \\
\hline Hipótesis nula & Eigenvalue & $\begin{array}{l}\text { Traza } \\
\text { estad }\end{array}$ & $\begin{array}{c}\text { Valor Crítico } \\
0.05\end{array}$ & Prob. \\
\hline Ninguna & 0.266698 & 14.33598 & 15.49471 & 0.0742 \\
\hline A lo más 1 & 0.030656 & 1.307687 & 3.841466 & 0.2528 \\
\hline
\end{tabular}

Fuente: cálculos propios en eviews 6.0

Tabla 3. Estimación para el consumo de HIP

\begin{tabular}{|c|c|c|c|c|}
\hline \multicolumn{5}{|c|}{ Variable Dependiente: DMCH } \\
\hline \multicolumn{5}{|c|}{ Método: MCO } \\
\hline \multicolumn{5}{|c|}{ Muestra: 2000-I 2010-IV } \\
\hline \multicolumn{5}{|c|}{ observaciones: 42 después de ajustado } \\
\hline $\begin{array}{l}\text { Variable } \\
\text { DMCH(-1) }\end{array}$ & $\begin{array}{c}\text { Coeficiente } \\
-0.244843 \\
\end{array}$ & $\begin{array}{r}\text { Desv. Estand } \\
1.32 \mathrm{E}-01 \\
\end{array}$ & $\begin{array}{l}\text { t-Estad } \\
-1.854192 \\
\end{array}$ & $\begin{array}{l}\text { Prob. } \\
0.0717 \\
\end{array}$ \\
\hline DMYD & 0.387382 & 0.067186 & 5.765798 & 0 \\
\hline DMYD(-1) & 0.245725 & 0.076379 & 3.217175 & 0.0027 \\
\hline DMA & -26.84468 & 15.0661 & -1.781793 & 0.083 \\
\hline DMCRED & 0.093274 & 0.026148 & 3.567079 & 0.001 \\
\hline R-cuadrado & 0.629495 & \multicolumn{2}{|c|}{ Media var depend } & 4.759157 \\
\hline R-cuadrado ajustado & 0.589441 & \multicolumn{2}{|c|}{ Desv. Estand var depend } & $4.88 \mathrm{E}+02$ \\
\hline Desv. Estand de reg & $3.13 \mathrm{E}+02$ & \multicolumn{2}{|c|}{ criterio Schwarz } & 14.64589 \\
\hline SRC & $3.62 \mathrm{E}+06$ & \multicolumn{2}{|c|}{ Durbin-Watson } & 2.477599 \\
\hline Prob. Jarque-Bera & 0.979551 & \multicolumn{2}{|c|}{ Breusch-Pagan-Godfrey } & 0.2331 \\
\hline Prueba de Klein & No Multicol & \multicolumn{2}{|c|}{ Test de Ramsey-Prob } & 0.7317 \\
\hline
\end{tabular}

Fuente: cálculos propios en eviews 6.0 
Los resultados se resumen en la Tabla 4:

Tabla 4. Resultados de los parámetros de la HIP

\begin{tabular}{|l|c|c|c|c|}
\hline Parámetro & $\delta$ & $\beta_{1}$ & $\beta_{2}$ & $\beta_{3}$ \\
\hline Estimación & 1.245 & 0.5085 & -26.84 & 0.093 \\
\hline
\end{tabular}

Fuente: cálculos propios.

La evidencia refleja que el grado de ajuste de las expectativas es de 1.245, es decir, en cada trimestre la esperanza de ajuste del consumo tiene una fuerte sobrerreacción, porque el consumo, inicialmente, tiene una gran reacción a las variables que se encuentren explicándolo, pero en los siguientes trimestres los agentes ajustan sus expectativas a lo sucedido. También se evidencia que la PMC a largo plazo es de 0.5085 , es decir, por cada peso que aumente el ingreso permanente, el consumo permanente aumenta en 0.508 pesos, osea que a largo plazo existe una mayor propensión marginal al ahorro que a corto plazo, ya que esta propensión permanente es de 0.4915 .

Por otra parte, la riqueza tiene un impacto negativo sobre el consumo a lo largo del tiempo, aunque en este punto dada la cointegración entre las series en niveles y la estacionariedad de las series transformadas se puede decir que los coeficientes a corto plazo pueden representar el coeficiente a largo plazo. Es por esta razón que por cada punto que aumenta el IPVU, el $\mathrm{CH}$ disminuye de un trimestre a otro en 26.84 miles de millones de pesos de 2005 .
Los resultados de la estimación reflejan que los colombianos a medida que son más ricos consumen menos en términos relativos, esto es, existe mayor propensión a ahorrar; lo que es cierto cuando se habla de la utilidad que le brinda consumir una unidad adicional de un bien cuando ya se está satisfecho. Finalmente, cuando se identifica el efecto de la restricción de liquidez, se muestra que por cada peso que aumenta el crédito el consumo se incremente en 0.093 .

En este sentido, al identificar los determinantes que explican el consumo mediante la HIP, es necesario señalar que los factores se asemejan a los mostrados por Hernández (2006), quien hizo un análisis acerca de los determinantes del consumo total de los hogares, utilizando diversas teorías económicas del consumo, y encontró que las variables que intervienen en la explicación, para el caso colombiano, son la riqueza, la tasa de interés, el ingreso corriente y la dinámica del sistema financiero, es decir, pone en consideración la mayoría de las variables aquí descritas, aunque vistas globalmente.

En síntesis, se observa que los colombianos tienen una visión miope del 
futuro y sobrerreaccionan a las expectativas. Además, el crédito y la riqueza representan un papel importante dentro de las decisiones del gasto en consumo. Igualmente, lo que se evidencia a largo plazo es que existen condiciones que impulsarían el ahorro de los colombianos por los resultados que arroja la propensión marginal a ahorrar ${ }^{17}$.

A pesar de la funcionalidad del modelo, existen diversas críticas a su utilización, ya que según Fernández (2009), la HIP puede fallar desde tres puntos de vista: i) el empírico, porque las estimaciones predicen el consumo pero por debajo de su nivel real; ii) el econométrico, pues según Deaton (1992) los modelos macroeconómicos poseen los problemas tradicionales en las series de tiempo, como las regresiones espurias, elevados $\mathrm{R}$ cuadrados que se combinan con bajos Durbin-Watson y una inexistencia de pruebas de diagnóstico ${ }^{18}$; iii) se relaciona con las apreciaciones de Lucas (1976), quien señaló que con expectativas racionales pueda que no existan, las relaciones estructurales, y no se debía esperar una relación estable entre ingreso corriente y permanente, porque cualquier cambio en la economía puede afectar la óptica como los agentes infieren a partir del ingreso corriente su ingreso permanente ${ }^{19}$.
No obstante, la utilidad del método definido es considerable, ya que existen diversos estudios que desean explicar el consumo con una visión a largo plazo, y es el caso del realizado por Núñez (2009), que contrasta la HIP en las comunidades autónomas españolas para el periodo 1980-2003, con el fin de identificar si un choque en el ingreso ocasiona un ajuste en el consumo de igual tamaño en el ajuste hecho. El estudio de Núñez finaliza manifestando que los datos validan la hipótesis pero en un sentido débil.

Otro estudio que se ha hecho referente al tema es el de Arriaga (2009), quien mediante el uso del modelo de renta permanente estima el efecto de la revalorización de los activos inmobiliarios en España sobre el consumo, tomando el efecto riqueza como mecanismo de traspaso para el impacto. El estudio en mención demostró que los aumentos en la riqueza inmobiliaria incrementan la capacidad potencial de endeudamiento por el mayor valor del colateral; o bien, se reduce el ahorro para aumentar su consumo.

Desde el punto de vista latinoamericano existe un estudio en Uruguay de Echenique (s.f.), que estima un modelo de consumo a partir de ecuaciones de Euler que dieron como resultado que los

\footnotetext{
Se invita al lector a revisar el artículo de López et al. (1996), quienes hablan sobre la caída de la tasa de ahorro en Colombia en los noventa.

18 Aunque este problema se reduce con la metodología utilizada.

19 No obstante, desde el punto de vista econométrico, los dos primeros reparos son subsanables, pero el problema es en el tercer factor, donde no existe certeza de las expectativas racionales de los agentes dentro de la economía colombiana.
} 
consumidores no tienen una trayectoria de consumo, por tanto existe una inestabilidad en el comporta-miento de este.

\section{Hipótesis del ciclo vital (HCV)}

\subsection{Revisión teórica}

En 1954, Franco Modigliani propuso la hipótesis del ciclo vital (HCV). Señaló, al igual que Friedman, que el consumo depende de las expectativas que tengan los consumidores sobre sus ingresos futuros y no solo del ingreso del período actual. Además, ambos autores comparten que los agentes maximizan su utilidad a lo largo de su vida teniendo en cuenta su visión sobre el futuro.

Sin embargo, Modigliani se distancia de la HIP en la medida que la $\mathrm{HCV}$ considera que de acuerdo con el ciclo de vida por el que esté atravesando el individuo, este tiene una consideración diferente respecto al ahorro ${ }^{20}$. Otra diferencia es que Modigliani, al contrario de Friedman, priorizó su análisis sobre la evolución del ciclo de vida del consumo familiar y del ingreso, y además identificó que la familia posee una vida limitada, es decir, analizó todo el proceso que tiene un individuo desde el momento en que empieza su vida laboral hasta cuando la termina -reflejada en la jubilación-. Los supuestos desarrollados por Modigliani (1986), desde el análisis de $\mathrm{HCV}$, son:
- Existe un conocimiento perfecto del mercado.

- Existe una función de utilidad homogénea.

- No hay ningún motivo de legado.

- Mercados de capital perfectos.

- Todas las unidades familiares de la economía poseen las mismas funciones de utilidad y utilizan las mismas tasas de descuento.

- Es constante la distribución por edad, por edad del ingreso, y por edad del patrimonio neto.

- El ingreso esperado es proporcional al ingreso corriente.

- La incertidumbre con respecto a las expectativas de las remuneraciones futuras no afectan a la asignación del consumo.

- El horizonte de planeación es toda la duración de la vida.

- La tasa de preferencia temporal es constante.

- Las acciones del individuo se ajustan a sus planes de consumo de toda la vida.

De los supuestos anteriores, Modigliani llegó a la siguiente ecuación:

$$
\begin{aligned}
C_{\mathrm{t}}= & \beta_{1}+\beta_{2} y d_{\mathrm{t}}+\beta_{3} y d_{\mathrm{t}}^{\mathrm{e}} \\
& +\beta_{4} A_{\mathrm{t}-1}+\beta_{5} r_{\mathrm{t}}
\end{aligned}
$$

En la ecuación (15) se evidencia que el consumo depende del ingreso corriente $\left(y d_{\mathrm{t}}\right)$, del ingreso disponible esperado $\left(y d^{\mathrm{e}}{ }_{\mathrm{t}}\right)$, de la riqueza neta del periodo anterior $\left(A_{\mathrm{t}-1}\right)$, y de la tasa de interés $\left(r_{\mathrm{t}}\right)$.

$\overline{20}$ Así, por ejemplo, cuando el individuo empieza su vida laboral, ahorra de tal manera que cuando se jubile desahorre. 
Aquí el concepto de riqueza de Modigliani se diferencia del de Friedman, ya que este último toma la riqueza en proporción al ingreso, y en la $\mathrm{HCV}$ se toma la riqueza neta. En definitiva, Modigliani (1986) argumentó diversas implicaciones de su teoría, entre las cuales se encuentran:

- La tasa de ahorro de un país es totalmente independiente del ingreso percápita.

- Diferentes tasas de ahorro nacional son consistentes con un comportamiento semejante del ciclo de vida de cada persona.

- Si se comparan países con comportamientos individuales idénticos, la tasa de ahorro agregada será mayor en la medida en que aumenta la tasa de crecimiento a largo plazo de la economía.

- Si el crecimiento económico es igual a cero, la tasa de ahorro será igual a cero.

- El coeficiente ingreso-riqueza es una función decreciente de la tasa de crecimiento, de tal forma que se alcanza el punto más alto en un crecimiento igual a cero.

- Una economía puede acumular un stock de riqueza substancial respecto al ingreso, incluso si no hay riqueza que se transmita por medio de legados.

- El parámetro principal que controla el coeficiente ingreso-riqueza y la tasa de ahorro para un crecimiento dado es la duración predominante del retiro.
Todo lo anterior justifica los efectos de las tasas de interés y de las decisiones de las familias según su visión sobre el futuro. No obstante, algunos autores como Lorenzo y Osimani (2001) toman como variables relevantes en esta teoría la distribución por edades en toda la economía. Estos toman el promedio de edad de los integrantes del hogar para explicar el ahorro en las familias de menores ingresos en Uruguay. Finalmente, cabe indicar que existen otras variables como la edad de retiro, comúnmente utilizada en estudios relacionados con pensiones; y la esperanza de vida, que se utiliza para evaluar variables sociales que definen el desarrollo en un país.

\subsection{Estimación econométrica}

Los datos necesarios para la estimación son el $\mathrm{CH}$, el YD, la riqueza (A), la restricción a la liquidez CRED, y los rendimientos de los activos financieros en el mercado(RM). Esta última se construyó con base en el comportamiento de la Bolsa de Valores para Colombia, que para el caso, se tomaron los datos del Índice de la Bolsa de Bogotá entre el primer trimestre del 2000 hasta el segundo trimestre de 2001, y del IGBC entre el tercer trimestre de 2001 y cuarto trimestre de $2010^{21}$. La explicación teórica surge por la existencia de una hipótesis acerca del impacto de la Bolsa sobre el consumo ${ }^{22}$; sin embargo, en esta

\footnotetext{
$21 \quad$ La explicación para tomar de ese modo los datos se sustenta en el hecho de que antes del tercer trimestre de 2001, las bolsas de valores existentes en el país no se habían integrado.

22 En EE.UU. Karen y Dean (2001) hacen estimaciones de este efecto.
} 
sección se asume que el rendimiento en el mercado de valores es la tasa de interés que los consumidores identifican cuando hacen su decisión de consumo intertemporal.

Para calcular el rendimiento real RM se toman los rendimientos nominales por trimestre y se le descuenta el promedio del IPC. Ahora, para estimar la HCV es fundamental establecer que las variables estén cointegradas, estando ya el $\mathrm{CH}$, la A y el CRED; falta por identificar si existe cointegración entre los rendimientos y el $\mathrm{CH}$ en niveles, la cual queda reflejada en la Tabla 5 que muestra la existencia de no más de una relación de equilibrio a largo plazo entre estas variables.

Tabla 5. Prueba de cointegración para $\mathrm{CH}$ yRM

\begin{tabular}{|c|c|c|c|c|}
\hline \multicolumn{5}{|c|}{ PRUEBA DE JOHANSEN } \\
\hline \multicolumn{5}{|c|}{ Tendencia Lineal Determinística } \\
\hline \multicolumn{5}{|c|}{ SERIES: CH, RM } \\
\hline \multicolumn{5}{|c|}{ Muestra: 2000-I 2010-IV } \\
\hline \multicolumn{5}{|c|}{ observaciones: 42 después de ajustado } \\
\hline Hipótesis nula & Eigenvalue & $\begin{array}{l}\text { Traza } \\
\text { estad }\end{array}$ & $\begin{array}{c}\text { Valor Crítico } \\
0.05\end{array}$ & Prob. \\
\hline Ninguna & 0.376877 & 20.89656 & 15.49471 & 0.0069 \\
\hline A lo más 1 & 0.024227 & 1.030077 & 3.841466 & 0.3101 \\
\hline
\end{tabular}

Fuente: cálculos propios en eviews 6.0

Tabla 6. Estimación para el ahorro de HCV

\begin{tabular}{|c|c|c|c|c|}
\hline \multicolumn{5}{|c|}{ Variable Dependiente: DMS } \\
\hline \multicolumn{5}{|c|}{ Método: MCO } \\
\hline \multicolumn{5}{|c|}{ Muestra: 2000-I 2010-IV } \\
\hline \multicolumn{5}{|c|}{ observaciones: 42 después de ajustado } \\
\hline Variable & Coeficiente & Desv. Estand & t-Estad & Prob. \\
\hline DMYD & 0.610364 & 6.92E-02 & 8.822685 & 0.0000 \\
\hline DMYD(-1) & -0.213819 & 0.070431 & -3.035872 & 0.0045 \\
\hline $\mathrm{RM}(-4)$ & 7.14224 & 3.716389 & 1.921822 & 0.0628 \\
\hline DMA & 27.44074 & 15.43184 & 1.77819 & 0.0841 \\
\hline DMCRED & -0.07929 & 0.026144 & -3.032861 & 0.0045 \\
\hline R-cuadrado & 0.799943 & \multicolumn{2}{|c|}{ Media var depend. } & 16.7 \\
\hline R-cuadrado ajustado & 0.777079 & \multicolumn{2}{|c|}{ Desv. Estand var depend. } & $6.76 \mathrm{E}+02$ \\
\hline Desv. Estand de reg & $3.19 \mathrm{E}+02$ & \multicolumn{2}{|c|}{ criterio Schwarz } & 14.69809 \\
\hline SRC & $3.57 \mathrm{E}+06$ & \multicolumn{2}{|c|}{ Durbin-Watson } & 2.473892 \\
\hline Prob. Jarque-Bera & 0.99683 & \multicolumn{2}{|c|}{ Breusch-Pagan-Godfrey } & 0.2355 \\
\hline
\end{tabular}

Fuente: cálculos propios en eviews 6.0 
Fundamentado en la ecuación (15), se estima con MCO el ahorro según la HCV, y se establece que la mejor ecuación utilizando la metodología de Blanchard (2003) es la siguiente:

$$
\begin{aligned}
D M S_{\mathrm{t}} & =\beta_{1} D M Y D_{\mathrm{t}}+\beta_{2} D M Y D_{\mathrm{t}-1}+\beta_{3} R M_{\mathrm{t}-4} \\
& +\beta_{4} D M A_{\mathrm{t}}+\beta_{5} D M C R E D_{\mathrm{t}}+\mu_{\mathrm{t}}
\end{aligned}
$$

La ecuación (16) muestra la propensión a ahorrar, el efecto que tiene el rendimiento del mercado sobre el ahorro un año después de la variación, y el efecto de la restricción de la liquidez. La Tabla 6 muestra que en el modelo se cumplen todos los supuestos ya indicados. Además, se muestra que el ajuste es bastante alto, ya que las variables exógenas explican cerca de $80 \%$ el comportamiento del ahorro.

En la Tabla 6 se observa que la PMA es de 0.3965 , esto es, que por cada peso que aumenta el ingreso disponible, los colombianos ahorran 0.39 pesos, y el resto lo dedican al consumo. También se muestra que la relación entre los rendimientos del mercado y el ahorro es positiva, ya que cuando se den las posibilidades de consumir en el futuro una mayor porción de bienes, los agentes se encuentran dispuestos a ahorrar mayor cantidad de sus ingresos, siendo en
Colombia que por cada $1 \%$ que aumenta la rentabilidad en el mercado, un año después el ahorro aumenta en 7.14 miles de millones de pesos de 2005 .

Por el lado de la riqueza se evidencia, lo contrario a lo que afirma la teoría ${ }^{23}$, que por cada punto que aumenta el precio de la vivienda, el ahorro aumenta en 27.44 miles de millones de pesos de 2005 , porque los agentes ahorran cuando poseen mayores volúmenes de riqueza. Finalmente, el efecto de la restricción de liquidez desde el crédito sobre el ahorro es negativo, debido a que por cada peso que aumenta el crédito, los agentes deciden disminuir su ahorro vital en 0.079 pesos, porque esto les garantizaría que sus posiciones de gasto no se vean truncadas cuando el ahorro disponible no sea suficiente. Por tanto, los hogares en Colombia deciden ahorrar más cuando su ingreso corriente aumenta, los rendimientos de los activos financieros aumentan, el valor de la riqueza es mayor, y los créditos son más flexibles.

\section{HIPÓTESIS DE HALL}

\subsection{Revisión teórica}

Dicha hipótesis también se conoce como la hipótesis del ingreso permanente con expectativas racionales $^{24}$ (HIPER). Esta

\footnotetext{
23 López y Salamanca (2009) estimaron el efecto riqueza de la vivienda en Colombia, donde las estimaciones arrojaron que la vivienda como elemento que representa la riqueza afecta al consumo, con una propensión marginal a consumir de 0.012 , que significa que por cada $1 \%$ que aumenta la riqueza, el consumo se incrementaría en el valor señalado. La diferencia de los resultados se encuentra en la metodología de estimación, porque los autores señalados utilizaron un modelo de equilibrio general dinámico.

24 Ver Muth (1961) quien desarrolló la hipótesis de las expectativas racionales.
} 
fue propuesta por Robert Hall (1978), basándose en la enérgica crítica que hizo Robert Lucas acerca de la teoría convencional del consumo. En principio, Hall agregó al modelo del ingreso permanente el supuesto de las expectativas racionales, lo cual hace que la incertidumbre juegue un papel preponderante en el comportamiento del consumo (Fernández, 2009).

De hecho, Clarida (1991) señala que la idea de Hall estaría indicando que si la teoría del ingreso permanente es cierta, y los consumidores tienen expectativas racionales, entonces el consumo debería seguir una caminata aleatoria, así las variaciones en el consumo son impredecibles. En este sentido, este modelo manifiesta que solo los cambios no anticipados en la riqueza y en el ingreso varían el ingreso permanente, razón por la cual se afecta el consumo en alguna medida que no es imaginable, es decir los cambios en el consumo no son predecibles (Blanchard \& Fischer, 2006). Este hecho es una contundente conclusión en el modelo de Hall porque estaría señalando que el uso de políticas económicas, al contrario de Keynes, es incierto (Romer, 1996).

Por otra parte, la idea central de este modelo es que los agentes basan su consumo de acuerdo al ingreso futuro esperado. Sin embargo, como éste es impredecible o incierto el problema del consumidor viene a ser la incertidumbre (Romer, 1996). Es por eso que Hall critica los anteriores modelos, y hace que el consumo siga un proceso auto regresivo de orden uno y analiza el caso concreto de la función de utilidad cuadrática.

Antes de decir cómo se resuelve un problema de consumo a la manera de Hall, hay que tener en cuenta cinco supuestos claves para este autor: i) la tasa de interés es constante para todo t, ii) la tasa de descuento es igual a cero, iii) la función de utilidad es cuadrática, iv) todos los agentes conocen todos los aspectos del modelo, y v) todas las variables estocásticas se mantienen invariables en el tiempo (Romer, 1996). El problema para Hall es el siguiente:

$\operatorname{Max} E\left\{\sum_{j=0}^{T} \beta^{\mathrm{j}} U\left(C_{\mathrm{t}}\right)-V\left(l_{\mathrm{t}+\mathrm{j}}\right)\right\}$

sujeto a $A_{\mathrm{t}-1}=\left(1+r_{\mathrm{t}}\right) A_{\mathrm{t}}+w_{\mathrm{t}} l_{\mathrm{t}}-C_{\mathrm{t}}$

Para resolver este problema multiperiodos se acude a la ecuación de Bellman que convierte este problema en dos periodos de la siguiente manera:

$V(A t)=\operatorname{Max} U\left(C_{\mathrm{t}}\right)-V\left(l_{\mathrm{t}}\right)+\beta E_{\mathrm{t}} V\left(\mathrm{~A}_{\mathrm{t}+1}\right)$

$+\lambda\left[\left(1+r_{\mathrm{t}}\right) A_{\mathrm{t}}+w_{\mathrm{t}} l_{\mathrm{t}}-C_{\mathrm{t}}-A_{\mathrm{t}+1}\right]$

Donde, $C_{\mathrm{t}}$ representa el consumo en el periodo t, $L$ es el trabajo en el periodo t, $A_{\mathrm{t}}$ representa la riqueza neta en el periodo $\mathrm{t}, r_{\mathrm{t}}$ representa la tasa de interés real en el periodo $\mathrm{t}, w_{\mathrm{t}}$ es el salario en el momento t, $A_{\mathrm{t}+1}$ representa la riqueza neta en el periodo $t+1$, y finalmente al incluir el operador matemático $E_{\mathrm{t}}$ se está 
incluyendo el supuesto de expectativas racionales (Sargent, 1978) ${ }^{25}$.

De la ecuación (19) se obtienen las condiciones de primer orden, es decir se deriva respecto a:

Variable Control $C_{\mathrm{t}}$ :

$$
U^{\prime}\left[\left(C_{\mathrm{t}}\right)\right]=\lambda_{\mathrm{t}}
$$

Variable control $l_{\mathrm{t}}$ :

$$
V^{\prime}\left[\left(l_{\mathrm{t}}\right)\right]=w_{\mathrm{t}} \lambda_{\mathrm{t}}
$$

Variable Estado $A_{\mathrm{t}}$ :

$$
V^{\prime}\left(A_{\mathrm{t}}\right)=\left[\left(1+r_{\mathrm{t}}\right]_{\mathrm{t}}\right.
$$

Variable Estado-Control $A_{\mathrm{t}+1}$ :

$$
E_{\mathrm{t}}\left[V^{\prime}\left(A_{\mathrm{t}+1}\right)\right]=\lambda_{\mathrm{t}}
$$

Restricción:

$$
A_{\mathrm{t}+1}=\left(1+r_{\mathrm{t}}\right) A_{\mathrm{t}}+\mathrm{w}_{\mathrm{t}} \mathrm{l}_{\mathrm{t}}-C_{\mathrm{t}}
$$

Una vez obtenidas las condiciones de primer orden, se procede a obtener la tasa marginal de sustitución entre el trabajo y el consumo. Para ello, se combinan las ecuaciones (21) y (22) y se tiene:

$* \quad \frac{V^{\prime}\left(l_{\mathrm{t}}\right)}{U^{\prime}\left(C_{\mathrm{t}}\right)}=\frac{w_{\mathrm{t}} \lambda_{\mathrm{t}}}{\lambda_{\mathrm{t}}}$
Eliminando $\lambda_{\mathrm{t}}$ se tiene:

$$
\frac{V^{\prime}\left(l_{\mathrm{t}}\right)}{U^{\prime}\left(C_{\mathrm{t}}\right)}=w_{\mathrm{t}}
$$

Por otra parte, la idea es llegar a la ecuación de Euler, que para este caso se quiere ver la relación entre el consumo presente y el futuro, a través de la utilidad marginal de cada periodo (Romer, 1996). En ese sentido, se adelanta un periodo la ecuación (22) y se tiene:

$$
V^{\prime}\left(A_{\mathrm{t}+1}\right)=\left(1+r_{\mathrm{t}+1}\right) \lambda_{\mathrm{t}+1}
$$

Esta ecuación se remplaza en (23) y se obtiene:

$$
\beta E_{\mathrm{t}}\left[\left(1+r_{\mathrm{t}+1}\right) \lambda_{\mathrm{t}+1}\right]=\lambda_{\mathrm{t}}
$$

Como $U^{\prime}\left(C_{\mathrm{t}}\right)=\lambda_{\mathrm{t}} \quad \mathrm{y} \quad U^{\prime}\left(C_{\mathrm{t}+1}\right)=\lambda_{\mathrm{t}+1}$ entonces remplazando estos valores en la anterior ecuación se obtiene la ecuación de Euler, que fue la ecuación que introdujo Hall:

$$
\beta E_{\mathrm{t}}\left[\left(1+r_{\mathrm{t}+1}\right) U^{\prime}\left(C_{\mathrm{t}+1}\right)\right]=U^{\prime}\left(C_{\mathrm{t}}\right)
$$

Hall (1978) respecto a esta ecuación supone que la tasa de interés es constante para todo t y que $\beta\left(1+r_{t+1}\right)=1$, por ende la ecuación se simplifica a:

$$
E_{\mathrm{t}}\left[U^{\prime}\left(C_{\mathrm{t}+1}\right)\right]=U^{\prime}\left(C_{\mathrm{t}}\right)
$$

\footnotetext{
$\overline{25}$ El lector puede consultar el artículo de Sargent (1978) que habla sobre las expectativas racionales en torno al consumo.
} 
Y de esta ecuación, Hall supone una función de utilidad cuadrática de la forma

$U\left(C_{\mathrm{t}}\right)=a C_{\mathrm{t}}-b C_{\mathrm{t}}^{2}$

Derivando respecto al consumo se tienen las siguientes ecuaciones:

$U^{\prime}\left(C_{\mathrm{t}}\right)=a-2 b C_{\mathrm{t}}$

$U^{\prime}\left(C_{\mathrm{t}+1}\right)=a-2 b C_{\mathrm{t}+1}$

Remplazando (32) y (33) en (31) se tiene:

$E_{\mathrm{t}}\left[a-2 b C_{\mathrm{t}+1}\right]=a-2 b C_{\mathrm{t}}$

Resolviendo esta ecuación se llega a

$E_{\mathrm{t}}\left(C_{\mathrm{t}+1}\right)=C_{\mathrm{t}}$

Y resolviendo el valor esperado se obtiene

$$
C_{\mathrm{t}+1}=C_{\mathrm{t}}+\varepsilon_{\mathrm{t}+1}
$$

De ahí que se diga que el consumo sigue una caminata aleatoria ${ }^{26}$, razón por la cual para Hall el uso de políticas es incierto. Por eso, Hall (1978) señala que la mejor predicción que se puede tener del consumo en el periodo $t+1$ o futuro, es el consumo alcanzado en el presente o en el periodo t. Lo valioso del término de error $\varepsilon_{t+1}$ es que recoge los sucesos impredecibles ocurridos en el consumo entre el periodo $t$ hasta $t+1$.
El hecho de que Hall suponga expectativas racionales implica dos momentos. Por un lado, en el que se forma la expectativa, es decir, la creencia en que un suceso puede ocurrir dada una probabilidad, la cual está sujeta a la ocurrencia de algunos eventos. En ese sentido, el agente determina el consumo en el presente dada la expectativa que tiene respecto al ingreso permanente del mismo momento.

Asimismo, señala que el agente utiliza toda la información disponible a su alcance para modificar su comportamiento de consumo en el periodo futuro, porque estima que su ingreso permanente futuro se modificará por algunos sucesos. Este hecho implica que el agente esté enterado de los movimientos que se presentan en ciertas variables económicas, con el fin de hacer unas nuevas expectativas que le permitan adaptarse a los posibles cambios, que desde su propia perspectiva, van a suceder si y solo si suceden los eventos que el agente cree que sucederán. En síntesis, el agente modificará su comportamiento de consumo si se dispone de nueva información económica.

\subsection{Estimación econométrica}

Para efectuar la estimación econométrica de la hipótesis de Hall, es necesario tomar los datos de la DMCH calculada por Blanchard (2003), con el propósito de identificar si el consumo de los hogares

Ante la aseveración de Hall, acerca de que el consumo sigue una caminata aleatoria, Deaton (1992) señala que como
la ecuación (36) no manifiesta información sobre la varianza de , la ecuación no es una caminata aleatoria porque no se puede suponer que la varianza del error sea constante. 
en Colombia responde a una caminata aleatoria, y si es efectivamente definido por un componente autorregresivo y un componente de error. Por tanto, antes de empezar a hacer las estimaciones es necesario mostrar el comportamiento de $\mathrm{DMCH}$ en el periodo muestral (ver Gráfico 1).

Gráfico 1. Comportamiento de DMCH

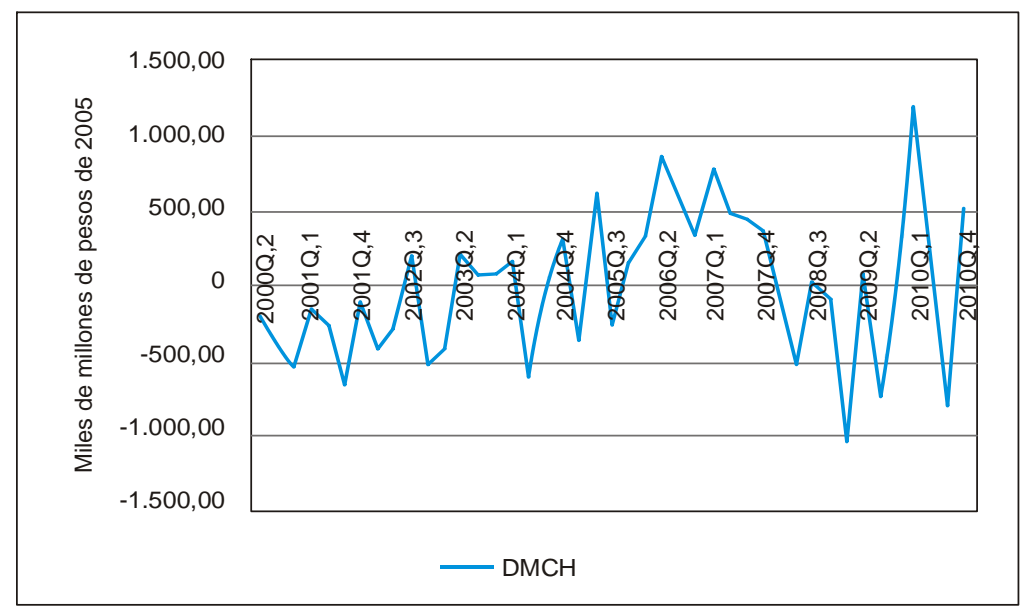

Fuente: cálculos propios con la metodología de Blanchard (2003)

El Gráfico 1 muestra el comportamiento volátil del crecimiento del consumo de los hogares, el cual es más fuerte durante los periodos de tensión económica, es o sea, en situaciones como la crisis de los EE.UU. en el 2008 que coincidió con la política monetaria contractiva que precipitó un descenso en los niveles de consumo. En el Gráfico 1 es posible notar la existencia de un patrón de comportamiento, pero antes de asegurar esto es necesario realizar las pruebas que podrían llevar a consolidar la explicación del consumo mediante la hipótesis de Hall.
El planteamiento de Hall define que el único factor que explica el consumo es este mismo en forma rezagada, por lo cual es necesario encontrar los patrones de comportamiento mediante la evaluación de un ARIMA. Lo primero que se requiere es identificar la estacionariedad de la serie mediante la prueba de raíz unitaria de Dickey-Fuller aumentada, donde la serie es efectivamente estacionaria. Es necesario aclarar que la serie tomada no está en niveles, ya que tuvo una transformación anterior. Un segundo aspecto es la identificación de los rezagos que se obtuvo mediante el 
correlograma en eviews 6.0, del cual se deduce que el mejor rezago que puede pronosticar el consumo es el tercero. Por tanto, la ecuación que puede sustentar la hipótesis de Hall es la siguiente:

$$
D M C H_{\mathrm{t}}=\beta_{1} A R(3)+\mu_{\mathrm{t}}
$$

La ecuación (37) indica el proceso autorregresivo óptimo para estimar las condiciones de la caminata aleatoria del consumo. En la Tabla 7 se muestra el cumplimiento de los supuestos de no autocorrelación, homoscedasticidad, no multicolinealidad, normalidad y correcta especificación del modelo. Además, se evidencia que el coeficiente de determinación es de $15.68 \%$, que al compararlo con las anteriores estimaciones es notablemente inferior.

Tabla 7. Estimación del DMCH con la hipótesis de Hall

\begin{tabular}{|c|c|c|c|c|}
\hline \multicolumn{5}{|c|}{ Variable Dependiente: DLNCH } \\
\hline \multicolumn{5}{|c|}{$\begin{array}{l}\text { Método: } \text { MCO } \\
\end{array}$} \\
\hline \multicolumn{5}{|c|}{ Muestra: 2000-I 2010-IV } \\
\hline \multicolumn{5}{|c|}{ observaciones: 42 después de ajustado } \\
\hline Variable & Coeficiente & Desv. Estand & t-Estad & Prob. \\
\hline $\mathrm{AR}(3)$ & 0.410702 & 0.150825 & 2.723037 & 0.0096 \\
\hline R-cuadrado & 0.156823 & \multicolumn{2}{|c|}{ Media var depend. } & 0.009239 \\
\hline R-cuadrado ajustado & 0.156823 & \multicolumn{2}{|c|}{ Desv. Estand var depend. } & $7.88 \mathrm{E}-03$ \\
\hline Desv. Estand de reg & $4.48 \mathrm{E}+02$ & \multicolumn{2}{|c|}{ criterio Schwarz } & -6.938737 \\
\hline SRC & $7.82 \mathrm{E}+06$ & \multicolumn{2}{|c|}{ Durbin-Watson } & 1.677832 \\
\hline Prob. Jarque-Bera & 0.87708 & \multicolumn{2}{|c|}{$\mathrm{ARCH}$} & 0.9753 \\
\hline Prueba de Klein & NA & \multicolumn{2}{|c|}{ Breusch-Godfrey Serial } & 0.6472 \\
\hline
\end{tabular}

Nota: NA significa en este caso no aplica.

Fuente: cálculos propios en eviews 6.0

En los resultados se muestra que cuando el consumo aumenta en un peso dentro de tres trimestres, este aumenta en 0.41 pesos, lo cual refleja únicamente un patrón y con poca explicación (ver R-cuadrado). Es por esta razón que en Colombia se nota que no hay una fuerte evidencia, por lo menos en sentido estadístico, sobre la aplicabilidad de la HIPER, debido a que las expectativas adaptativas (ver hipótesis HIP) parecen ser más significativas que las expectativas racionales. Lo anterior se prueba comparando los coeficientes de determinación de la función keynesiana y de la HIP de Friedman frente al de Hall, donde efectivamente este último es bastante inferior y no sirve para efectos de pronóstico.

En ese sentido, la hipótesis de Hall ha tenido varios detractores debido a que esta contradice la visión de que el consumo es 
predecible. Entre ellos se encuentran Mankiw (1982) y Mankiw y Cambell (1989), quienes trabajaron con datos agregados para los Estados Unidos y mostraron que la tesis de Hall no es definitiva, por lo que no se puede rechazar la visión que sostiene que los valores pasados del consumo y del ingreso ayudan a predecir el comportamiento del consumo $^{27}$ (Romer, 1996).

Otra crítica proviene de Shea (1995b), quien hizo una regresión del crecimiento del consumo en función del crecimiento esperado del salario. Shea quería contrastar si el coeficiente de la regresión daba cero o diferente de cero, puesto que si daba el primer resultado, según la tesis del ingreso permanente, la hipótesis de Hall se cumpliría. Sin embargo, el resultado fue de 0.89 , así para Shea el consumo no sigue una caminata aleatoria porque los hogares se enfrentan a restricciones de liquidez ${ }^{28}$, por lo cual estos acuden al ahorro para reducir las fluctuaciones del consumo (Romer, 1996). En síntesis, el modelo de caminata aleatoria del consumo no brinda la misma explicación que sí brindan modelos como el de Friedman y la función keynesiana, por lo que sería necesario plantear que para el análisis a largo plazo el modelo fundamental es el de Friedman y para choques a corto plazo es la función keynesiana de consumo.

\section{Conclusiones}

Este documento llevó a cabo las estimaciones econométricas de las hipótesis de consumo de Keynes, Friedman, Modigliani y Hall, para Colombia, con datos trimestrales, entre el periodo 2000 al 2010. Se debe indicar que todas las estimaciones que se hicieron se basaron en la metodología de Blanchard (2003), que comprende tres pasos: i) tomar la diferencia entre los datos de una variable (consumo), en determinado periodo -en este caso sería la diferencia entre trimestres-; ii) luego a los datos de estas diferencias se les saca el promedio; y iii) el promedio obtenido se utiliza para restarlo a cada diferencia del paso ii.

La ventaja de utilizar esta metodología reside en aislar el efecto de los incrementos tendenciales, que permite que las estimaciones obtenidas no sobrestimen los verdaderos parámetros. Además, cabe indicar que la metodología señalada requiere que las estimaciones no incluyan intercepto, porque este recoge los efectos de la tendencia y como lo que se quiere hacer es aislar el efecto que pueda tener esta sobre las estimaciones, la inclusión

\footnotetext{
Estos autores, según Romer (1996), descubrieron que la evolución de los precios de las acciones ayudan a predecir las variaciones del consumo.

28 También hay otros aspectos que merecen la pena ser analizados, tales como el ahorro precautorio, el de existencias reguladoras (Carroll, 1992, 1997, 2001), que es una extensión del sistema de ahorro precautorio (Leland (1968), Sadmo (1970), Kimball (1990)) la hipótesis de cuasi-racionalidad con la actualización de las decisiones de consumo e información imperfecta (Allen y Carroll, 2001);Akerlof y Yellen (1985) y Pischke (1995); y las condiciones de consumo con restricciones de liquidez (Hayashi, 1985) Fernández-Corugedo, 2002); entre otros modelos adicionales. Sin embargo, este texto no los abordará.
} 
del intercepto iría en contravía de lo que se desea. Por tanto, al utilizar la misma metodología para todas las estimaciones se obtiene una ventaja que radica en que estas se hacen comparables, no solo por el uso de MCO sino también por el cumplimiento de todos los supuestos que impone $\mathrm{MCO}$, lo cual hace que los estimadores sean útiles como insumos de medidas de política económica.

Los principales resultados del documento señalan que para el caso de la función de consumo keynesiana, la PMC estimada para Colombia en el periodo muestral fue de 0.62 , pero se debe anotar que el efecto que tuvo una variación del ingreso disponible (YD) en un peso provocó que el consumo agregado de los hogares $(\mathrm{CH})$ respondiera en el mismo periodo en 0.38 pesos y en el siguiente trimestre en 0.24 pesos. Esto estaría señalando que en su conjunto la propensión marginal a ahorrar (PMA), en Colombia, es de 0.38 pesos. Esta cifra señala, además, que un aumento de un peso del YD provocó que el ahorro respondiera en el mismo periodo en 0.23 pesos y en el siguiente trimestre en 0.15 pesos.

Respecto a los resultados de la hipótesis del ingreso permanente (HIP) se obtuvo que el coeficiente de expectativas fue de 1.245 , lo que viene a significar que los colombianos formaron sus pronósticos sobre el futuro más inmediato observando el pasado. Así ante aumentos del consumo del trimestre anterior, los colombianos sobrerreacionaron al incrementar su consumo en $24.5 \%$ por encima de lo esperado, lo que lleva luego a que las expectativas se corrijan en este porcentaje. La PMC a largo plazo fue de 0.508, lo cual significa que la PMA a largo plazo fue de 0.4915. Esto refleja que a largo plazo se presenta una mayor PMA que a corto plazo, lo que haría pensar que a largo plazo la acumulación de capital pueda financiarse. La riqueza tuvo un impacto negativo sobre el consumo, puesto que por cada punto que aumentó esta, el $\mathrm{CH}$ se redujo de un trimestre a otro en 26.84 miles de millones de pesos de 2005. Y se evidenció que aumentos en el crédito para el sector privado (CRED) provocan un efecto sobre el $\mathrm{CH}$ de 0.093 .

En cuanto a la hipótesis del ciclo vital (HCV), se nota que la PMA es de casi 0.4, y 0.6 viene a ser la PMC. Se observó que un incremento de $1 \%$ en los rendimientos del mercado el ahorro aumenta en 7.14 miles de millones de pesos, pero solo un año después.

En relación con la riqueza, se aprecia que por cada punto que aumenta el precio de la vivienda, el ahorro crece en 27.44 miles de millones de pesos. También se evidenció que aumentos en el CRED provocan un descenso sobre el $\mathrm{CH}$ de 0.079. Finalmente, cabe señalar que dado que el ajuste de bondad del ahorro está cerca de $80 \%$, esto viene a significar que en Colombia el ahorro es más predecible, que el consumo.

Respecto a la hipótesis del ingreso permanente con expectativas racionales (HIPER), formulada por Hall, los 
resultados mostraron que cuando el consumo de tres trimestres anteriores aumenta en 1 peso, el consumo actual se incrementa en 0.41 pesos. El resultado obtenido reflejó que la HIPER tiene poco grado explicativo para Colombia, lo cual viene a significar que son las expectativas adaptativas las que parecen ajustarse en mayor medida al comportamiento del consumo, en vez de las expectativas racionales.

Finalmente, la función de consumo keynesiana suministra la PMC (0.62) y la PMA (0.38) a corto plazo; mientras que la HIP provee la PMC $(0.508)$ y la PMA a largo plazo (0.491). Esto permite señalar que en Colombia se satisface la conjetura según la cual a medida que crece el ingreso en el tiempo, los agentes dedicarán una menor proporción hacia el consumo; en consecuencia, la parte del ingreso que se dedicaría hacia el ahorro sería mayor, resultado contrario al que encontraron Hernández y Chahín (2008), quienes señalaron que la tasa de ahorro permanece constante en el tiempo para Colombia. Por tanto, se puede argüir que en materia de política económica los resultados en este documento señalarían, por un lado, que a largo plazo la acumulación de capital podría financiarse con el ahorro de largo plazo, que a su vez provocaría un estímulo para el crecimiento económico; y por el otro, una tasa de ahorro mayor podría traducirse en una reducción de la demanda agregada.

No obstante, saber cuál es el efecto que perdurará es ambiguo, ya que depende de múltiples factores tales como microeconómicos, macroeconómicos, entre otros. En este sentido, quedan preguntas sin resolver que podrían ser objeto de estudio en otros documentos, por ejemplo, las cuestiones relacionadas con el impacto de la PMA a corto y a largo plazo sobre el crecimiento económico; junto con el efecto amortiguador que representaría el ahorro dentro de las fluctuaciones que podría sufrir el consumo en un determinado periodo.

\section{R eferencias bibliográficas}

1. Allen, T. W. \& Carroll, C. D.(Abril, 2001). Individual learning about consumption. Macroeconomic Dynamics, 5(02),255-271.

2. Akerlof, G. A. \&Yellen, Y. L. (Septiembre, 1985). Can small deviations from rationality make significant differences to economic equilibria.TheAmerican EconomicReview, American EconomicAssociation75 (4), 708720.
3. Arriaga, R.(Primer cuatrimestre de 2009). Efecto riqueza a partir de la revalorización de los activos inmobiliarios: análisis y evidencia empírica para el caso español. Análisis Económico,Universidad Autónoma Metropolitana México, XXIV(55),7-28.

4. Blanchard, O. (2003). Macroeconomía. (3ra. Ed.). Cap. 4. Buenos Aires: PrenticeHall Press. 
5. Blanchard, O. \& Fischer, S. (1993). Lectures on macroeconomics. Cambridge, Massachusetts: The MIT Press.

6. Briceño, A. y Camelo, M. (2011). Análisis macroeconómico del Plan Nacional de Desarrollo, 2010-2014 "Prosperidad para todos": aplicación de un modelo simple de demanda. Universidad Católica de Colombia.

7. Cagan P. (1956). The monetary dynamics of hyperinflation.En Studies in the quantity. Theory of money. (pp. 25-117). Chicago: Milton Friedman.

8. Carroll, C.D.(1992).The buffer stock theory of saving: some macroeconomic evidence. Brookings paper on economic activity, (2).

9. Carroll, C.D. (1997).Buffer stock saving and the life cycle permanent income hypothesis. The quarterly journal of economics, CXII.NBER Working Papers Cambridge, MA., (5788).

10. Carroll, C.D. (Abril, 2001). Precautionary saving and the marginal propensity to consume out of permanent income. NBER Working PapersCambridge, MA.,(8233).

11. Clarida, R. H.(1991).Aggregate stochastic implications of the life-cycle hypothesis. MIT Quarterly Journal of Economics, 106.

12. Deaton, A.(1992). Understanding consumption. New Jersey: Princeton University, Clarendon Press. Publishedto Oxford Scholarship Online.

13. Echenique, F.(1996).La teoría del consumo, un análisis empírico para datos de Uruguay: estimación de ecuaciones de Euler. Revista de Economía, Banco Central del Uruguay, III (1).

14. Fernández, E. (2009). Teoría del Consumo. Ensayos. Centro de estudios monetarios latinoamericanos CEMLA,(77).
15. Fernández, C. W. (2002). Soft liquidity constraints and precautionary saving.Bank of England, Working paper (158).

16. Flavin, M. (1985).Excess sensitivity of consumption to current income: liquidity constrains or myopia?Canada Journal of Economics, University of British Columbia, 18,117-36.

17. Gross, D. \& Nicholas, S. (2001).Do liquidity constraints and interest rates matter for consumer behavior? Evidence from credit card data.The Quarterly Journal of Economics, NBER Working papers(8314).

18. Hall, R. (1978).Stochastic implications of the life cycle-permanent income hypothesis: theory and evidence, Journal of Political Economy, University of Chicago, 86.

19. Hansen, G. D.(1985).Indivisible labor and the business cycle.Journal of Monetary Economics, University of California, 16 (3),309-327.

20. Hayashi, F.(1985).The effect of liquidity constraints on consumption: a crosssectional analysis. NBER WorkingPapers (0882).

21. Hernández, A. J.(Diciembre, 2006). Revisión de los determinantes macroeconómicos del consumo total de los hogares para el caso colombiano. Revista ESPE, Banco de la República, (52).

22. Hernández, G.\&Chahín, D.(2008). Apuntes de teoría de consumo.Bogotá:Pontificia Universidad Javeriana, Facultad de Ciencias Económicas y Administrativas.

23. Karen, E. y Dean, M. (2001). Does stock market wealth matter for consumption. Brookings Institution, Board of Gobernors FED discussion paper, (23).

24. Keynes, J. M. (1936). Teoría general de la 
ocupación, el interés y el dinero. México: Fondo de CulturaEconómica.

25. Kimball, M. S. (1990). Precautionary saving in the small and in the large.NBER Working Paper (2848), 53-73.

26. Kirman, A. P. (1992). Whom or what does the representative individual represent? Journal of Economic Perspectives, 6(2), 117-136.

27. Leland, H. E.(1968).Saving and uncertainty: the precautionary demand.Quarterly journal of economics, 82, 465-473.

28. López, E. y Salamanca, A. (2009). El efecto de la riqueza de la vivienda en Colombia, Borradores de Economía, $\mathrm{N}^{\circ}$. 551, pp.1-58.

29. López, A; Gómez, C; Rodríguez, N. (1996). La ciada de la tasa de ahorro en Colombia durante los años noventa: evidencia a partir de una base de datos para el periodo 19501993.Borradores de Economía, $\mathrm{N}^{\circ}$. 57, pp. $1-42$.

30. Lorenzo, F. \&Osimani, R. (2001). Alternativas de política para fortalecer el ahorro de los hogares de menores ingresos: el caso de Uruguay. Santiago de Chile: ONU.

31. Lucas, R. (1976).Econometric policy evaluation: a critique.En Brunner\&Meltzer, A.The Phillips curve and labour markets. (pp. 257-284).North Holland: Journal Carnegie-Rochester Conference Series on Public Policy.

32. Mankiw, N. G.(1982). Hall's consumption hypothesis and durable goods.Journal of Monetary Economics, 10,417-425.

33. Mankiw, J. \& Campbell, N. (1989). Consumption income and interest rates: reinterpreting the time series evidence. NBER
Macroeconomic Annual, 185-246.

34. Modigliani, F. (1986). Life cycle, individual trift, and the wealth of nations.American economic review, 7, 297-313.

35. Muth, J. (Julio, 1961). Rational expectations and the theory of price movements. Econometrica, 29, (3), 315-335.

36. Pischke, J. (Julio, 1995). Individual income, incomplete information and aggregate consumption.Princeton University, WorkingPapers(669), 805-840.

37. Plan Nacional de Desarrollo 2010-2014, (2011).

38. Roca, R. (s.f.). Teorías del consumo y el ahorro. Macroeconomía Avanzada, Universidad Mayor de San Marcos y Pontificia Universidad Católica del Perú.

39. Roca, R.(2008).Las expectativas adaptativas y racionales y la dinámica macroeconómica. Macroeconomía Avanzada, Universidad Mayor de San Marcos y Pontificia Universidad Católica del Perú.

40. Romer, D. (1996). Advanced Macroeconomics. $\left(2^{\text {nd }}\right.$. Ed.). s.l.: McGraw.Hill.

41. Sadmo, A.(Julio, 1970). The effect of uncertainty on saving decisions. Review of economic studies, Vanderbilt University, 37, 353-360.

42. Sargent, T. (Agosto, 1978). Rational expectations, econometric exogeneity, and consumption. Journal of Political Economy, 86(4), 673-700.

43. Shea, J.(Marzo, 1995).Union contracts and the life-cycle/permanent-income hypothesis. American Economics Review, Vanderbilt University,85 (1),186-200. 